\title{
Towards evidence-based staffing: the promise and pitfalls of patient-to-intensivist ratios
}

\author{
Meeta Prasad Kerlin ${ }^{1 *}$ (id and Pedro Caruso 2,3
}

(c) 2022 Springer-Verlag GmbH Germany, part of Springer Nature

As coronavirus disease 2019 (COVID-19) cases surged at different times all over the world, news stories about running out of personal protective equipment, triaging ventilators, and mobile morgues have played on repeat. Likewise, human resources have been stretched beyond their limits. Those same news outlets tell stories of health care workers experiencing burnout and depression and posttraumatic stress disorder, of clinicians leaving their professions en masse [1]. While this is especially salient in the current moment, the intensivist workforce has been in crisis for years, and shortages are projected to worsen [2]. Therefore, understanding the tipping point of when the workload of an intensivist is too much, the point at which the quality of their care diminishes, is essential for intensive care unit (ICU) organization even beyond our current crisis.

A few studies have sought to quantify the optimal physician workload in different settings. Two studies of ICU patients in the United Kingdom (UK) and France demonstrated that above certain thresholds of patient load per intensivist, mortality was increased $[3,4]$. In the UK study, the relationship of patient volume with hospital mortality was U-shaped, with both lower and higher patient loads associated with higher hospital mortality. This relationship is consistent with a "just right" conceptual model-where too few patients lead to inefficiency and inexperience that is detrimental to care delivery, and too many lead to inability to provide timely and

\footnotetext{
*Correspondence: prasadm@pennmedicine.upenn.edu

${ }^{1}$ Department of Medicine, Perelman School of Medicine at the University of Pennsylvania, 423 Guardian Drive, 302 Blockley Hall, Philadelphia, PA 19103, USA

Full author information is available at the end of the article
}

appropriate care to all of them. However, another study in a United States population did not confirm an association of intensivist-to-ICU-bed ratio with mortality; on the other hand, this study was conducted in a single ICU that underwent a series of organizational changes and was also being staffed by fellow and resident physicians [5]. These inconsistencies highlight the complexity of critical care delivery. Prior studies have not rigorously accounted for other ICU organizational factors known to affect patient outcomes, notably other concurrent staffing models [6]. In this issue of Intensive Care Medicine, Gershengorn and colleagues aim to further expand the evidence around intensivist workload with a retrospective cohort study examining the relationship between one measure of workload-the patient-to-intensivist ratio (PIR) - and hospital mortality of critically ill patients [7]. Their study had several advantages over previous ones. They used a high-quality, granular database of virtually all ICU patients in Australia and New Zealand, with over 90,000 patients across 73 ICUs. They had not only patient data but also detailed staffing data; therefore, they were able to precisely estimate PIR, adjust for differences in patient case mix, and account for other ICU staffing, such as patient-to-nurse ratio. They employed sophisticated statistical methods and conducted several wellconsidered sensitivity analyses.

The authors had hypothesized that there would be a U-shaped relationship between the PIR and mortality, as observed previously [3]. However, despite carefully planned and robust sensitivity analyses, they found no such association. At first, we intensivists may ask how can this possibly be? Even before this pandemic, many or even all of us have experienced a time of having too many patients to deliver the best possible care, of knowing

\section{Springer}


that too much has been asked of us. And previous studies have indeed confirmed this fear, demonstrating that higher patient loads have consequences of inefficiencies and even patient harm. On deeper evaluation, however, perhaps we see that the result is not unexpected. The authors offered several possible explanations and acknowledged several limitations. The ICUs were lower acuity than most previous studies, thus with a lower percentage of patients who might actually benefit from critical care. Furthermore, the study ICUs may not be representative of ICUs more broadly. Besides being lower acuity, they also had a fairly small range of PIR, suggesting attention as already paid to intensivist workload. The ICUs were generally well-staffed, with a majority of intensivists having at least one junior doctor present to share the workload, and high availability of nursing resources other than the assigned bedside nurse. Therefore, the study may have limited generalizability to ICUs outside of Australia and New Zealand.

We feel that two key points bear further discussion. First, a particularly important question is the relevance of the simple definition of average PIR during ICU admission. Gershengorn and colleagues adopted the average of daily PIR as the key measure of intensivist workload. This choice was pragmatic; PIR is easily measured and easily understood. A threshold value for PIR, if identified, would have clear implications for staffing policies. However, we feel that the PIR is too simple. As the authors themselves acknowledge, it does not capture all the factors that influence intensivist workload, the construct at the heart of this research question. An average daily PIR does not allow for the possibility that interventions-and therefore strain-may have different impact at different points in a patient's illness. In large ICUs staffed by more than one intensivist concurrently, it does not account for the possibility that patient assignments may differ based on acuity or other factors. And of course, it cannot account for the overall acuity of the patients, which can dramatically affect the intensity and complexity of an intensivist's workload.

Another point made by the authors that we wish to highlight is that these results should not be taken to imply that intensivists can care for an unlimited number of patients. Patient mortality is but one outcome that is relevant to the question of optimal intensivist workload, and we must not rely too heavily on what is an incomplete picture of the whole story. This pandemic has brought into sharp focus the detrimental effects of strain on the critical care workforce. Professional burnout is peaking amongst our colleagues $[8,9]$, and clinicians of all professional groups are leaving in droves, compromising the size and quality of a workforce already in shortage. Instead, we might consider these results as a new perspective on this question in a new context. The finding that PIR was not associated with differences in mortality in ICUs that are low acuity, well-staffed, with standardized practices across large national health systems can be interpreted as a confirmation that non-intensivist factors and resources can support and expand capacity for caring for critically ill patients. Perhaps, this study can lead us to look beyond the PIR for solutions to meet the evergrowing demands for critical care.

\section{Author details \\ ${ }^{1}$ Department of Medicine, Perelman School of Medicine at the University of Pennsylvania, 423 Guardian Drive, 302 Blockley Hall, Philadelphia, PA 19103, USA. ${ }^{2}$ Intensive Care Unit, AC Camargo Cancer Center, São Paulo, Brazil. ${ }^{3}$ Pul- monary Division, Heart Institute (InCor), Hospital das Clínicas da Faculdade de Medicina da Universidade de São Paulo, São Paulo, Brazil.}

\section{Declarations}

Conflicts of interest

MPK and PC have no potential conflicts of interest to disclose.

\section{Publisher's Note}

Springer Nature remains neutral with regard to jurisdictional claims in published maps and institutional affiliations.

Accepted: 24 December 2021

Published online: 13 January 2022

\section{References}

1. Jacobs A (2021) 'Nursing is in crisis': staff shortages put patients at risk. N YorkTimes. 1:1

2. Kelley MA, Angus DC, Chalfin DB et al (2004) The critical care crisis in the United States: a report from the profession. Crit Care Med 32(5):1219-1222

3. Gershengorn HB, Harrison DA, Garland A, Wilcox ME, Rowan KM, Wunsch $H$ (2017) Association of intensive care unit patient-to-intensivist ratios with hospital mortality. JAMA Intern Med 177(3):388-396

4. Neuraz A, Guérin C, Payet C et al (2015) Patient mortality is associated with staff resources and workload in the ICU: a multicenter observational study. Crit Care Med 43(8):1587-1594

5. Dara SI, Afessa B (2005) Intensivist-to-bed ratio: association with outcomes in the medical ICU. Chest 128(2):567-572

6. Zampieri FG, Salluh Jl, Azevedo LC et al (2019) ICU staffing feature phenotypes and their relationship with patients' outcomes: an unsupervised machine learning analysis. Intensive Care Med 45(11):1599-1607

7. Gershengorn HB, Pilcher DV, Litton E, Anstey M, Garland A, Wunsch H (2021) Association of patient-to-intensivist ratio with hospital mortality in Australia and New Zealand. Intensive Care Med. https://doi.org/10.1007/ s00134-021-06575-z

8. Wahlster S, Sharma M, Lewis AK et al (2021) The coronavirus disease 2019 pandemic's effect on critical care resources and health-care providers: a global survey. Chest 159(2):619-633

9. Kerlin MP, Silvestri JA, Klaiman T, Gutsche JT, Jablonski J, Mikkelsen ME (2021) Critical care clinician wellness during the COVID-19 pandemic: a longitudinal analysis. Ann Am Thorac Soc. https://doi.org/10.1513/Annal SATS.202105-567RL 